\title{
Mode of lysozyme protein adsorption at end-tethered polyethylene oxide brushes on gold surfaces determined by neutron reflectivity
}

\author{
Warren Taylor $^{1, \text { a }}$, Stephen Ebbens ${ }^{1}$, Maximillian W.A. Skoda ${ }^{2}$, John R.P. Webster ${ }^{2}$, and Richard A.L. Jones ${ }^{1}$ \\ 1 Physics and Astronomy Department, University of Sheffield, Sheffield, UK \\ 2 ISIS, Rutherton Appleton Laboratory, Oxfordshire, UK
}

Received 19 January 2014 and Received in final form 20 August 2014

Published online: 9 March 2015

(c) The Author(s) 2015. This article is published with open access at Springerlink.com

\begin{abstract}
The mode of lysozyme protein adsorption at end-tethered thiol-terminated polyethylene oxide brushes grafted upon gold was determined in situ by neutron reflectivity using the INTER instrument at target station 2, ISIS, RAL, UK. It was found that the most probable position of protein adsorption at these weakly protein resistive brushes was at the gold-brush interface in the so-called primary protein position.
\end{abstract}

\section{Introduction}

Protein adsorption is believed to enable the adherence of micro-organisms which eventually form robust biofilms $[1,2]$. Biofilms are a hindrance to many applications such as the hulls of ships, process equipment, and various medical devices [3]. Once formed biofilms are very resilient and can be very difficult and costly to remove $[4$, 5]. In order to prevent biofilm formation much research has been conducted into producing coatings which resist the initial adsorption of protein. One approach, which has been found to be particularly effective at resisting protein adsorption, is to coat surfaces with end-tethered polyethylene oxide (PEO) polymers at high grafting densities such that the chains are overlapping and in the brush regime [619]. It has been found that adsorbed amount of protein at end-tethered PEO surfaces is dependent upon the grafting density and molecular weight of the chains, with variation between studies believed to be due to various factors such as distal chemistry or brush-substrate interactions. In previous work by the authors, Taylor and Jones, it was found that the adsorb amount of lysozyme protein at PEO brushes on gold decreases with increasing coverage, implying that PEO polymers essentially block the adsorption of protein at the substrate-brush interface [20]. The exact position of adsorbed protein at end-tethered polyethylene oxide brushes has long been a mystery and is of significant importance in understanding the antifouling properties of such surfaces. As of yet no study has been able to actually measure directly where protein is adsorbing at PEO poly-

\footnotetext{
a e-mail: 4.warren.taylor@gmail.com
}

mer brushes. Knowledge of where proteins are adsorbing will allow the confirmation of various theoretical work explaining the protein resistive mechanism of PEO polymer brushes.

Theoretical and experimental work often refer to the following three main adsorption positions: A) primary adsorption - at the brush substrate interface, B) secondary adsorption - at the brush liquid interface and C) tertiary adsorption - where protein adsorbs throughout the brush due to attractive interaction between the proteins and PEO chains, see fig. 1 and fig. 2. Various theories attempt to explain primary adsorption at PEO brushes, and take into account how insertion of protein affects the free energy of the brushes due to energetically unfavorable restrictions in chain conformation [21-27]. Most of these theories assume that PEO does not bind with proteins on the segmental level due to its charge neutrality, weak van der Waal interactions and strong hydrogen bonding with water. However, work conducted in the Leckband group has found that PEO brushes when under compression can become attractive towards streptavidin-proteincoated surfaces, stressing that PEO on the segmental level is not strictly non-protein binding [16,22,28,29]. Further evidence of attractive PEO-protein interactions have been observed via light scattering experiments conducted by Bloustine et al., were PEO in solution was actually found to have a weak affinity for lysozyme proteins, and speculated to be due to hydrogen bonding [30]. Molecular simulations have also shown that PEO will hydrogen bind with lysine peptides a common constituent of many proteins [31]. Therefore, the possibility of a tertiary or secondary adsorption of protein at PEO brushes has also 


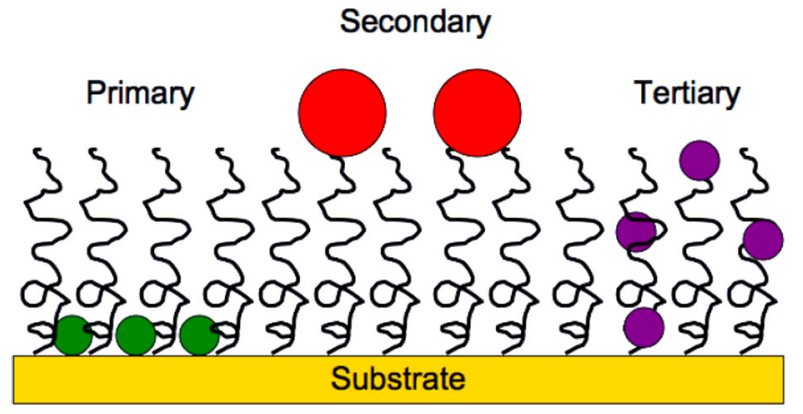

Fig. 1. Proposed modes of protein adsorption at a PEO brush; primary at the substrate, secondary at the edge of the brush and tertiary along the polymer chains.

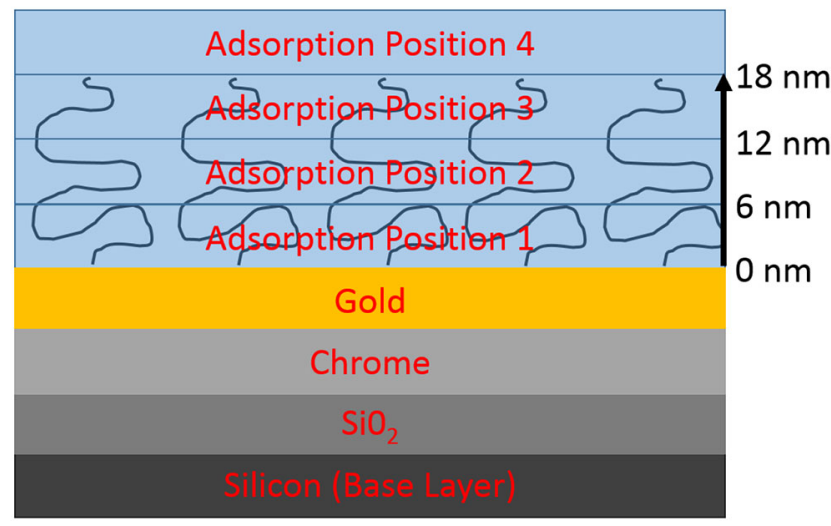

Fig. 2. Shows a graphical representation of the model in table 1 . Scale alongside shows distance each adsorption position is away from the gold/brush interface.

been proposed to explain these results, in particular theory work by Halperin et al. where an $n$-cluster type model is demonstrated $[32,33]$. It has also been found experimentally that methyl-PEG brushes become protein binding at high grafting density, whereas hydroxyl-PEG brushes do not, causing some to speculate a secondary adsorption at the edge of the polymer brush in the secondary position is possible, either promoted by the methyl end groups or conversely deterred by the hydroxyl groups [18,34], see fig. 1. Further studies have also stressed the importance of lateral fluctuations in surface coverage of polymer, and suggest that protein adsorption can mainly be accounted by primary adsorption upon bald spots where polymer is not present $[20,35]$.

Therefore there is a need to measure the exact position of protein adsorption in order to clarify and test the numerous explanations for the protein resistive mechanism of PEO brushes. Due to the innate difficulties in resolving matter at the length scales relevant to polymer brushes $\sim 10 \mathrm{~nm}$, up to now no study has yet been able to determine directly the position of the adsorbed protein in situ. However, neutron reflectivity does offer the possibility of resolving matter at these length scales and furthermore in situ. Due to the very small wavelength of neutrons, and the large contrast between the scattering length density of deuterated and hydrogenated materials, neutron reflectivity can measure the density profiles of similar systems down to angstroms of resolution $[36,37]$. Here we use neutron reflectivity collected using the INTER instrument at Target station 2 ISIS, RAL, Oxford to determine the most likely position that lysozyme proteins are adsorbing at weakly protein resistive brushes on well characterized polyethylene oxide brushes on gold.

In previous work we demonstrated a novel procedure for producing PEO polymer brushes on relatively smooth gold surfaces (roughness $\sim 2 \mathrm{~nm}$ ) [38]. We were able to accurately control the grafting of these brushes over the range of grafting at which $\mathrm{PEO}$ brushes become protein resistive using a concentrated homopolymer solution. In further work we showed that the adsorbed amount of protein at these surfaces follows an exponential decay with brush coverage [20]. We interpreted this relationship with a simple model that accounts for protein adsorption on the gold substrate in the primary position on areas not covered by polymer brush due to lateral fluctuations in the grafting of PEO chains. Here we use the same system and determine via neutron reflectivity that lysozyme protein is adsorbing in the primary position at two weakly protein resistive brushes.

\section{Experimental}

\section{Wafers and polymer brushes}

Two and a half inch wafers were purchased from Prolog Semicore. $3.5 \mathrm{~nm}$ of chrome and $26 \mathrm{~nm}$ of gold was deposited upon these surfaces by Vaculayer Ltd, Canada. These surfaces were found through AFM to be very smooth with an average roughness between samples of less than $1 \mathrm{~nm}$ (rms). The wafers were cleaned before use, via sonication in chloroform. Brushes were deposited via the concentrated homopolymer solution brush forming procedure described in previous work. In brief, PEO-SH chains at a low volume fraction $\sim 1 \%$ are dissolved in a concentrated solution of PEO and water. When the concentration of the $\mathrm{PEO}$ in the water solution is greater than $\sim 20 \%$, the PEO and PEO-SH chains have ideal chain statistics and can overlap in solution. Chain overlap allows the production of high density polymer brushes. By controlling the volume fraction and molecular weight of the PEO chains the grafting density of the PEO-SH chains can be precisely controlled. The thickness of brush layers was measured via ellipsometry before being placed in the neutron beam.

\section{Wet cell}

A wet cell designed by the ISIS team was used for the in situ neutron reflectivity experiments. This cell comprised of a 2 and a half inch silicon wafer which was placed below a test wafer. Between the two wafers was a $0.1 \mathrm{~mm}$ thick oring providing enough space for liquid. Such a thin chamber produced lamina flow, assuring a complete exchange of liquid. Two holes in the bottom wafer allow an inlet 
and outlet for the liquid. These holes where placed at either side of wafer maximizing liquid exchange. Liquid was exchanged within the cell by use of a hand syringe.

\section{Protein adsorption experimental methods}

An initial scan of the brush gold surface was taken in $\mathrm{D}_{2} \mathrm{O}$ ( $1 \mathrm{mM}$ Tris-HCL). Later $5 \mathrm{mg} / \mathrm{ml}$ of lysozyme in $\mathrm{D}_{2} \mathrm{O}$ was flushed through the chamber $(1 \mathrm{mM}$ Tris-HCL $p \mathrm{H} \sim 6.5)$. This protein solution was allowed to sit in the chamber for 20 minutes to allow the adsorption of protein. After 20 minutes the chamber was flushed with Tris-HCL buffer and a neutron scan taken of the wafer. The room was kept at a constant temperature of 24 degrees centigrade. After the experiment the wafers were removed, rinse-dried and re-measured via ellipsometry to determine an adsorbed protein dry thickness. A high concentration of lysozyme $5 \mathrm{mg} / \mathrm{ml}$ was chosen to assure that the protein adsorption reached close to maximum adsorption in 20 minutes. Preliminary QCM experiments, which will be published in future work, showed that adsorption process on goldand PEO-coated surfaces approximately reaches equilibrium after 20 minutes. The effect of changing the protein concentration is to change the kinetics of adsorption and adsorbed amount rather than changing the mode of protein adsorption at the PEO brushes.

\section{Neutron reflectivity}

Thermal neutrons have a distribution of wavelengths as low as $10^{-10} \mathrm{~m}$, and will scatter elastically off atomic nuclei. This means that NR is incredibly sensitive to the difference between the scattering length density of hydrogenated and deuterated materials. Therefore NR can detect the position of adsorbed protein at a surface immerged in $\mathrm{D}_{2} \mathrm{O}$. The change in neutron momentum $Q=$ $4 \pi \sin \Theta / \lambda$ is measured using time of flight and is plotted against neutron reflectivity. The INTER instrument at ISIS, RAL, Oxford, UK was used to collect the NR data presented here. The wavelength of neutrons used is between $1.5 \AA$ and $16 \AA$ and were incident upon the surface at an angle of 0.8 degrees. The raw neutron reflectivity data was normalized to set the sub-critical reflectivity to unity. Also, because of a slight misalignment of the detector heads, the $Q$ data of the recorded angle measurements required a rectifying adjustment of a factor of 1.02-1.03.

\section{Modeling of NR curves}

Modeling the NR curves using Motofit software enabled the most probable position of adsorbed material to be determined. An example of the models used to analyze the resulting NR curves is shown in table 1 . The values for the thicknesses of the individual layers in table 1 were found by fitting the layers with a genetic fit within the Motofit software. For surfaces with protein present the SLD of one of layers from 4 to 7 was modeled with the SLD of
Table 1. Motofit model of bare gold substrate. Layers 47 represent the different positions in which protein can adsorb. To represent primary adsorption (at $0 \mathrm{~nm}$ away from the gold/brush interface), layer four is given the SLD of 3.17 and the thickness and roughness is fitted. Whilst, to simulate secondary adsorption layer 7 is given a SLD of 3.17 whilst layers 4, 5 , and 6 are held at a SLD of 6.35 corresponding to that of the $\mathrm{PEO} / \mathrm{D}_{2} \mathrm{O}$ brush. Chi squared $\sim 4.83$, Model base roughness $\sim 0.5 \mathrm{~nm}, \mathrm{SLD}$ of $\mathrm{D}_{2} \mathrm{O} \sim 6.35\left(\cdot 10^{-6} \AA^{-2}\right)$, bkg $\sim 10^{-5}$.

\begin{tabular}{|l|l|l|l|}
\hline Layer & Thickness $(\mathrm{nm})$ & $\mathrm{SLD}\left(\cdot 10^{-6} \AA^{-2}\right)$ & $R g h(\mathrm{~nm})$ \\
\hline $1 \mathrm{SiO} 2$ & 3.3 & 3.47 & 0.5 \\
\hline $2 \mathrm{Ni}$ & 3.89 & 3.03 & 0.5 \\
\hline $3 \mathrm{Au}$ & 26.06 & 4.5 & 2.12 \\
\hline $4 \mathrm{Br} / \mathrm{Pr} / \mathrm{D}_{2} \mathrm{O}$ & 6 & 6.35 or 3.17 & 1 \\
\hline $5 \mathrm{Br} / \mathrm{Pr} / \mathrm{D}_{2} \mathrm{O}$ & 6 & 6.35 or 3.17 & 1 \\
\hline $6 \mathrm{Br} / \mathrm{Pr} / \mathrm{D}_{2} \mathrm{O}$ & 6 & 6.35 or 3.17 & 1 \\
\hline $7 \mathrm{Br} / \mathrm{Pr} / \mathrm{D}_{2} \mathrm{O}$ & 6 & 6.35 or 3.17 & 1 \\
\hline
\end{tabular}

lysozyme, $3.17 \cdot 10^{-6} \AA^{-2}$, instead of the SLD for $\mathrm{D}_{2} \mathrm{O}$ of $6.35 \cdot 10^{-6} \AA^{-2}$. Layers $4-7$ represent the 4 increasing distances away from the gold-brush interface at which the lysozyme protein was modeled to adsorb.

\section{Results and discussion}

Here the neutron reflectivity profiles for three PEO brush samples with dry thicknesses of $0 \mathrm{~nm}, 0.7 \mathrm{~nm}$ and $0.9 \mathrm{~nm}$ are shown before and after the adsorption of lysozyme protein, as seen in fig. 4. Four different models were fitted to the lysozyme NR curves representing adsorption at different distances away from the gold-brush interface, the chi squared and thicknesses of these layers can be seen in table 2. The ex situ dry thickness of lysozyme was measured by ellipsometry and are shown in table 2, all data will be referred to by their dry thickness as determined by ellipsometry here on in.

The experiments presented here are conducted on relatively complicated interfaces as there are three distinct layers in the bare gold-coated neutron wafers. As the number of layers at an interface increases the more difficult it becomes to resolve individual layers accurately. An added difficulty is that, for the experiment in hand, the volume fraction at which $20 \mathrm{kDa} M_{\mathrm{w}}$ PEO brushes change from being protein adsorbing to protein binding is very low. Typically $20 \mathrm{kDa} M_{\mathrm{w}}$ PEO brushes become protein resistant at volume fractions of around $3 \%$ and a brush of such a volume fraction has a SLD of $6.25 \times 10^{-6} \AA^{-2}$, which is very close to that of pure $\mathrm{D}_{2} \mathrm{O}$ at $6.35 \times 10^{-6} \AA^{-2}$. Therefore it is almost impossible to resolve such sparse and diffuse layers on the relatively complicated substrates used in these experiments. As can be seen in fig. 3, there is only a very small difference in neutron reflectivity profile for a bare gold surface and one which contains a polymer brush with an ellipsometry determined dry thickness of $0.9 \mathrm{~nm}$. Figure 3 also shows the predicted model NR curves for 


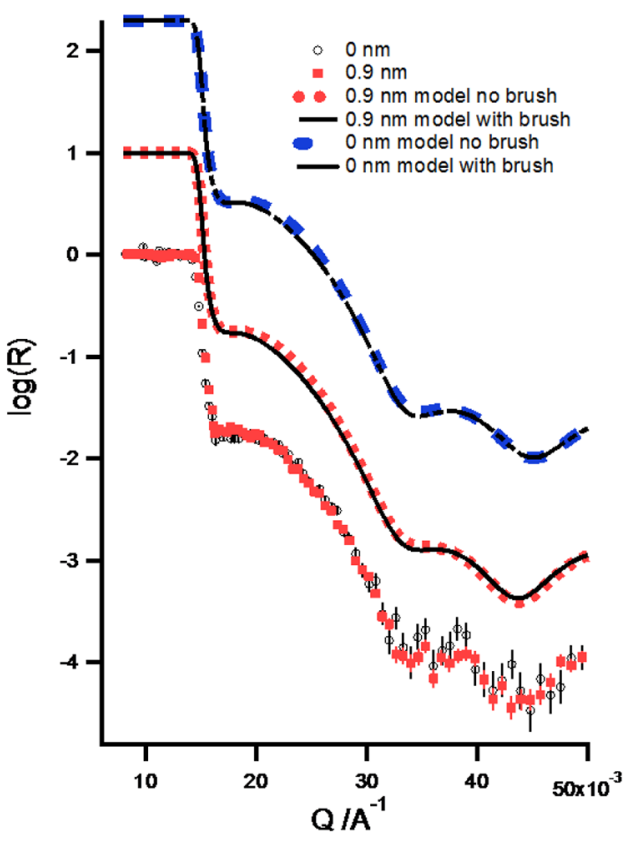

Fig. 3. Open circles: reflectivity profile for a bare gold surface in $\mathrm{D}_{2} \mathrm{O}$. Closed squares series is a gold surface with a $0.9 \mathrm{~nm}$ thick (as determined dry by ellipsometry) PEO polymer brush in $\mathrm{D}_{2} \mathrm{O}$. Red dashed line: model fit to $0.9 \mathrm{~nm}$ thick brush not taken into account the presence of a polymer brush. Solid line: the model fit for a swollen polymer brush with a vol frac of $3 \%$, SLD of $6.25 \cdot 10^{-6} \AA^{-2}$ and thickness $16 \mathrm{~nm}$. Blue dashed line: model fit to bare gold surface $0 \mathrm{~nm}$ brush. Solid line: the model fit for a swollen polymer brush with a vol frac of $3 \%$, SLD of $6.25 \cdot 10^{-6} \AA^{-2}$ and thickness $16 \mathrm{~nm}$ on top of this layer.

the $0 \mathrm{~nm}$ and $0.9 \mathrm{~nm}$ surfaces with and without a $16 \mathrm{~nm}$ swollen PEO brush with a SLD of $6.25 \times 10^{-6} \AA^{-2}$. It is clear that the curves are hardly different with or without the brush. Therefore, neutron reflectivity is ineffective at determining any reliable information about the density profile of such thin brush surfaces. However, there is a large contrast between the scattering length density of a relatively dense globular protein such as lysozyme (SLD $\sim 3.17 \times 10^{-6} \AA^{-2}$ ) [39] making its presence at the $0,0.7$ and $0.9 \mathrm{~nm}$ brush interfaces noticeable via a small change in the reflectivity, fig. 4 . Therefore it is possible to effectively treat the brush as if it were invisible and use neutron reflectivity to determine the location of the adsorbed lysozyme protein. Thus the acquired NR data should allow us to determine the most likely protein adsorption mode at PEO brushes.

Initially NR scans where taken for each surface submerged in $\mathrm{D}_{2} \mathrm{O}$ without protein in order to measure the thickness of the underlying metal layers; the NR curves and model fits for these brush surfaces can be seen in fig. 4 . Then the surfaces were measured via NR again after protein adsorption, the curves can also be seen in fig. 4 . In order to ascertain the protein adsorption position, a model was implemented that involved four separate slabs, which were initially set to have a scattering length density of $6.35 \cdot 10^{-6} \AA^{-2}$ and a thickness of $6 \mathrm{~nm}$, as seen in table 1 .
The refractive index of one of the layers from 4 to 7 was then set to the SLD of lysozyme i.e. $3 \cdot 17 \cdot 10^{-6} \AA^{-2}$, the remaining slabs had their SLD maintained at $6.35 \cdot 10^{-6} \AA^{-2}$. The thickness and roughness of the lysozyme slab were fitted using Motofit to produce the best fits for this adsorption distance away from the gold-brush interface. The Chi squared for these fits and modelled layer thicknesses were noted and compared to determine which basic mode of adsorption provided the best fits to the data, see table 2 . It was found that for the adsorption of lysozyme the lowest Chi square's were achieved for protein adsorbing in the 1st position, with the fits becoming significantly less good after a distance of $6 \mathrm{~nm}$. There is a slight decrease seen in Chi squared from 2nd to 3rd and then 4th position however this decrease is considerably smaller than the increase in Chi squared from the 1st to 2nd position. Also this slight decrease in Chi squared across the 2nd to 3rd position is also seen in the bare gold sample implying that this feature is there within the model regardless of the presence of a brush.

From table 2 it can be seen that the NR predicted thickness roughly gives a value $1 \mathrm{~nm}$ for the protein thickness for each sample even though the ellipsometry measured thickness show that the protein thickness is dropping from $1.8 \mathrm{~nm}$ to $0.9 \mathrm{~nm}$ with increasing brush thickness. Therefore it can be concluded that the NR experimental data and model here can only roughly predict the thickness of the protein layer. However the model analysis presented here is very sensitive to the distance the protein is adsorbing away from the gold substrate. The model produces unrealistically thin protein layers for adsorption in the 2nd, 3rd and 4th position. For instance, adsorption at the edge of the brush (4th position) for the $0.9 \mathrm{~nm}$ dry brush layer results in a model-determined thickness of $5.4 \times 10^{-6} \mathrm{~nm}$, clearly unrealistically thin. These very thin modeled layers further support the assertion that the protein is not adsorbing in these positions as the modeling software is driving the best fit solution towards one with a vanishingly thin adsorbed layer. Figure 4(a) and (b) show the best fits to the NR data showing that for all three surfaces the protein is adsorbing at the gold-brush interface, in the so-called primary position.

\section{Conclusion}

The adsorption of lysozyme upon relatively complicated substrates, consisting of silicon wafers coated with chrome and gold, was successfully detected using neutron reflectivity. Thiol-terminated PEO brushes were deposited upon these surfaces using the concentrated homopolymer solution procedure described in previous work [38]. These brush surfaces could be determined ex situ using ellipsometry, however due to their extremely low wet volume fraction and inherent complexity of the substrates used it was not possible to determine the presence of a hydrated brush using NR. These brush surfaces were subsequently exposed to solutions containing lysozyme protein. The NR curves before and after exposure to lysozyme were noticeably different for several of these surfaces. A model was 


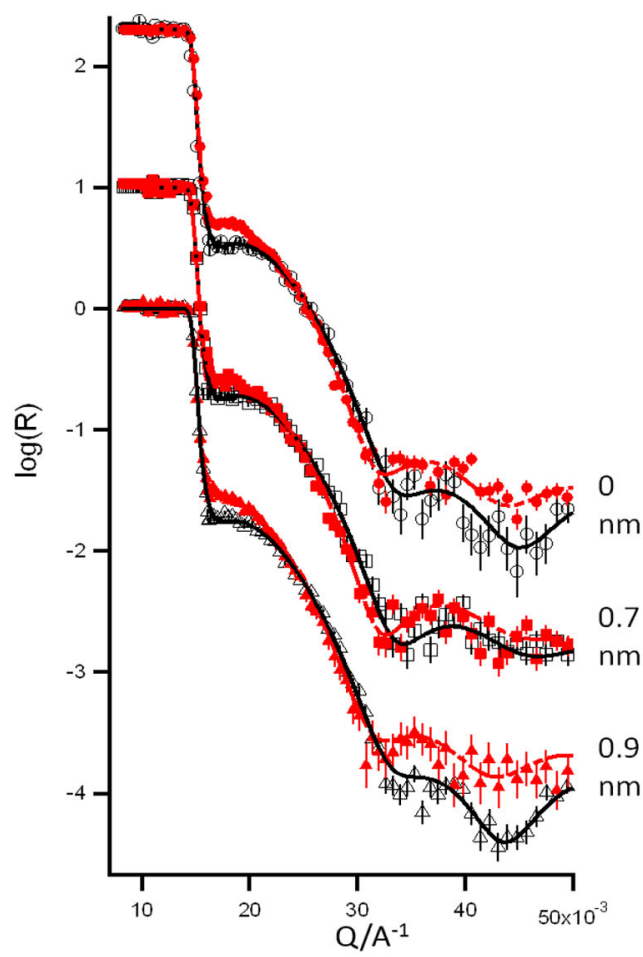

(a)

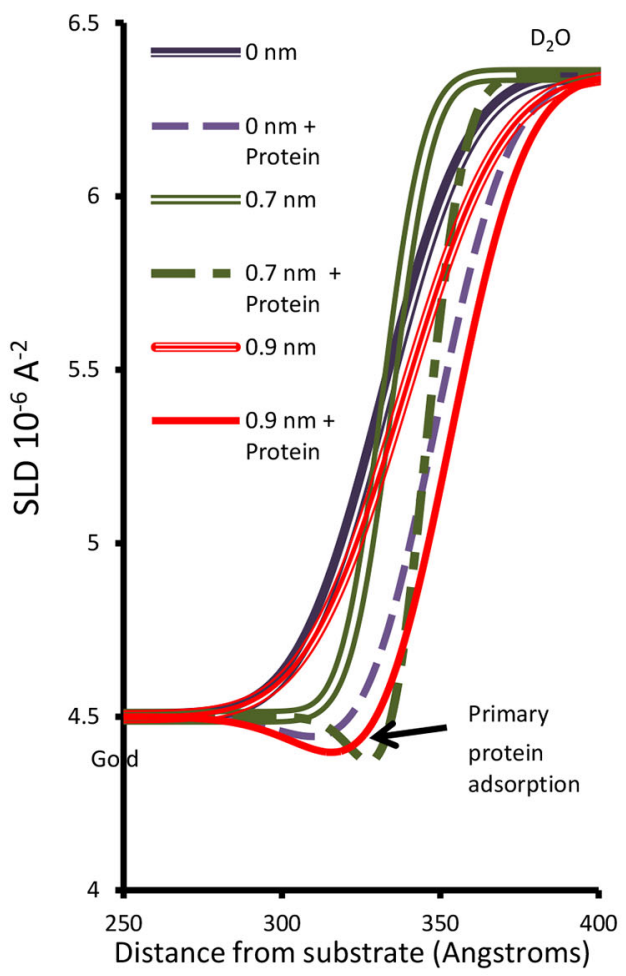

(b)

Fig. 4. NR curve (a) and SLD profile (b) for $0.7 \mathrm{~nm} 20 \mathrm{kDA}$ brush on gold with and without protein. Anotation for (a): Open and closed circles are $0 \mathrm{~nm}$ PEO brushes with and without protein, respectively; open and closed squares are $0.7 \mathrm{~nm}$ PEO brush with and without protein respectively; open and closed triangles are $0.9 \mathrm{~nm}$ PEO brush with and without protein respectively. Solid and dashed lines are model fits to data.

Table 2. Chi squared for fitted data for protein adsorption at the four different positions away from the surfaces: $0 \mathrm{~nm} \sim 1 \mathrm{st}$, $6 \mathrm{~nm} \sim 2 \mathrm{nd}, 12 \mathrm{~nm} \sim 3 \mathrm{rd}, 18 \mathrm{~nm} \sim 4 \mathrm{th}$, for the three different thicknesses of brush. The thickness of the protein layer is given next to the Chi squared for each layer. Edge of brush for $0.7 \mathrm{~nm}$ brush is $15 \mathrm{~nm}$ and for $0.9 \mathrm{~nm}$ is $16 \mathrm{~nm}$. Brush thickness along with adsorbed thickness of lysozyme protein is measured via ellipsometry before and after NR measurement, respectively.

\begin{tabular}{|c|c|c|c|c|c|c|c|c|c|}
\hline \multirow{2}{*}{ Ellip thickness (nm) } & \multicolumn{5}{|c|}{ Protein adsorption position: distance away from gold substrate. Determined by NR. } \\
\hline \multirow{2}{*}{ Brush } & \multirow{2}{*}{ Protein } & 1st position $(0 \mathrm{~nm})$ & 2nd position $(6 \mathrm{~nm})$ & \multicolumn{2}{|c|}{ 3rd position $(12 \mathrm{~nm})$} & \multicolumn{2}{c|}{4 th position $(18 \mathrm{~nm})$} \\
\cline { 3 - 10 } & & Chi & Th $(\mathrm{nm})$ & Chi & Th $(\mathrm{nm})$ & Chi & Th $(\mathrm{nm})$ & Chi & Th $(\mathrm{nm})$ \\
\hline 0 & 1.8 & 3.5 & 1.16 & 13.0 & $4 \times 10^{-5}$ & 12.2 & $3 \times 10^{-4}$ & 11.9 & $5 \times 10^{-5}$ \\
\hline 0.7 & 1.2 & 6.13 & 0.91 & 8.9 & $7 \times 10^{-4}$ & 8.6 & $1.7 \times 10^{-4}$ & 8.9 & $4.2 \times 10^{-5}$ \\
\hline 0.9 & 0.7 & 0.9 & 1.19 & 3.3 & $4.7 \times 10^{-6}$ & 3.2 & 0.037 & 2.9 & $5.4 \times 10^{-6}$ \\
\hline
\end{tabular}

used to determine how far away the protein is adsorbed at these surfaces. The model consisted of four different adsorption positions: 1 st at $0 \mathrm{~nm}, 2 \mathrm{nd}$ at $6 \mathrm{~nm}, 3 \mathrm{rd}$ at $12 \mathrm{~nm}$ and 4 th at $18 \mathrm{~nm}$ away from the substrate. It was found that, for all the layers, a best fit to the data was achieved for adsorption directly at the surface of the brush, with the goodness of fit becoming significantly worse assuming adsorption further away from the brush. This supports the assertion that lysozyme adsorption at these surfaces is at the gold substrate in the primary position. 
We would like to thank the EPSRC and Whiterose DTC for funding this project. We would also like to thank Michael P. Weir, Andrew Parnell, Tao Wang, and Hiroshi Hamamatsu for help during neutron reflectivity experiments, which was greatly appreciated.

Open Access This is an open access article distributed under the terms of the Creative Commons Attribution License (http://creativecommons.org/licenses/by/4.0), which permits unrestricted use, distribution, and reproduction in any medium, provided the original work is properly cited.

\section{References}

1. D. Pavithra, M. Doble, Biomed. Mater. 3, 13 (2008).

2. W. Norde, Z. Phys. Chem.-Int. J. Res. Phys. Chem. Chem. Phys. 221, 47 (2007).

3. K.E. Cooksey, B. Wigglesworthcooksey, Aquatic Microb. Ecol. 9, 87 (1995).

4. P.S. Stewart, J.W. Costerton, Lancet 358, 135 (2001).

5. A.K. Epstein et al., Proc. Natl. Acad. Sci. U.S.A. 108, 995 (2011).

6. J.G. Archambault, J.L. Brash, Colloids Surf. B 39, 9 (2004).

7. J.G. Archambault, J.L. Brash, Colloids Surf. B 33, 111 (2004).

8. H. Chen et al., Colloids Surf. B 61, 237 (2008).

9. H. Chen et al., Biomaterials 26, 2391 (2005).

10. X. Chen, R. Pelton, in Aicam 2005, edited by M. Nogami et al. (Trans Tech Publications Ltd, Zurich-Uetikon, 2006) pp. 363-366.

11. C. Czeslik, Z. Phys. Chem.-Int. J. Res. Phys. Chem. Chem. Phys. 218, 771 (2004).

12. F. Fang, I. Szleifer, Proc. Natl. Acad. Sci. U.S.A. 103, 5769 (2006).

13. J. Genzer et al., Langmuir 28, 2122 (2012).

14. S. Gon et al., Macromolecules 45, 8373 (2012).
15. P. Kingshott, H. Thissen, H.J. Griesser, Biomaterials 23, 2043 (2002).

16. D. Leckband, S. Sheth, A. Halperin, J. Biomater. Sci. Polym. Ed. 10, 1125 (1999).

17. W. Norde, D. Gage, Langmuir 20, 4162 (2004).

18. L.D. Unsworth, H. Sheardown, J.L. Brash, Biomaterials 26, 5927 (2005).

19. L.D. Unsworth et al., J. Colloid Interface Sci. 281, 112 (2005).

20. W. Taylor, R.A.L. Jones, Langmuir 29, 6116 (2013).

21. F. Fang, J. Satulovsky, I. Szleifer, Biophys. J. 89, 1516 (2005).

22. A. Halperin, D.E. Leckband, C. R. Acad. Sci. Paris, Ser. IV Phys. Astrophys. 1, 1171 (2000).

23. J. Satulovsky, M.A. Carignano, I. Szleifer, Proc. Natl. Acad. Sci. U.S.A. 97, 9037 (2000).

24. I. Szleifer, Physica A 244, 370 (1997).

25. I. Szleifer, Biophys. J. 72, 595 (1997).

26. S.I. Jeon et al., J. Colloid Interface Sci. 142, 149 (1991).

27. S.I. Jeon, J.D. Andrade, J. Colloid Interface Sci. 142, 159 (1991).

28. N.V. Efremova, S.R. Sheth, D.E. Leckband, Langmuir 17, 7628 (2001).

29. S.R. Sheth, D. Leckband, Proc. Natl. Acad. Sci. U.S.A. 94, 8399 (1997).

30. J. Bloustine et al., Phys. Rev. Lett. 96, 087803 (2006).

31. A. Jain, H.S. Ashbaugh, Biomacromolecules 12, 2729 (2011).

32. A. Halperin, Eur. Phys. J. B 3, 359 (1998).

33. A. Halperin et al., Langmuir 23, 10603 (2007).

34. L.D. Unsworth, H. Sheardown, J.L. Brash, Langmuir 24, 1924 (2008).

35. P. Katira, A. Agarwal, H. Hess, Adv. Mater. 21, 1599 (2009).

36. M.P. Weir et al., Langmuir 27, 11000 (2011).

37. S.M. Kilbey, J.F. Ankner, Curr. Opin. Colloid Interface Sci. 17, 83 (2012).

38. W. Taylor, R.A.L. Jones, Langmuir 26, 13954 (2010).

39. Y.M. Efimova et al., J. Radioanal. Nucl. Chem. 264, 271 (2005). 